\title{
Maturation of the Host Response and its Impact upon Periodontal Disease
}

\author{
Sidney H. Stein* and Anastasios Karydis \\ Department of Periodontology, UTHSC-College of Dentistry, 875 Union Avenue, Memphis, TN 38163, USA
}

Received: June 19, 2015; Accepted: July 07, 2015; Published: July 15, 2015

*Corresponding author: Sidney H. Stein, Department of Periodontology, UTHSC-College of Dentistry, 875 Union Avenue, Memphis, TN 38163, USA, Tel: +901-448-6242; Fax: +901-448-6751; E-Mail: sstein@uthsc.edu

\begin{abstract}
Absract
Periodontal disease is initiated by the accumulation of bacterial plaque on the tooth surface. Yet, disease progression is regulated by the local immune response in the periodontium. Therefore, disease susceptibility is determined by a variety of factors which modulate the host response such as genetics and the local environment within the oral cavity. The host response to the bacterial plaque in the gingival sulcus grows as the biofilm develops and matures. Initially, it will be predominated by neutrophils and innate immunity. If the host response is unable to contain the initial bacterial infection, the adaptive arm of the immune system will be activated. A classic histological study which described the sequence of cellular events following the accumulation of bacterial plaque was reported by Page and Schroeder in the late 70's. T lymphocytes were implicated as being critical for the evolution of the lesion from its early to its established phase. Subsequently, it was reported that most of the T lymphocytes were CD4+ cells, else Th1 or Th2. With the recent discovery of additional $\mathrm{T}$ helper cell subsets, evidence is accumulating to support a prominent role for Th17 cells and their cytokines in periodontal disease pathogenesis. In addition, the evolving pathogenic biofilm supports the outgrowth of a "keystone" bacterium, Porphyromonas gingivalis, which possess virulence factors that can inactivate critical elements of the host response and enhance the proliferation and differentiation of Th17 cells. The polymicrobial dysbiosis promotes an environment conducive for the outgrowth of pathogenic bacteria. This mini-review will explore how the Page and Schroeder model and the maturation of the host response have evolved over the past forty years. Furthermore, it will touch upon some host response modifiers that may play an important role as adjunctive aids in the individualization of periodontal therapy in the $21^{\text {st }}$ century.
\end{abstract}

Keywords: Host response; Th17; Polymicrobial dysbiosis; $P$. gingivalis; Periodontal disease

\section{Introduction}

\section{Page and Schroeder Model}

Periodontal disease is initiated by the accumulation of bacterial plaque in the biofilm, which forms on the surface of the epithelial cells and hard tissue. Disease susceptibility is determined by a variety of factors which regulate the individual host response such as genetics and the local environment within the oral cavity. The classic study by Page and Schroeder demonstrated that, the progression of periodontal disease from gingivitis to periodontitis can be loosely divided into a series of stages [1]. The initial lesion occurs within the first four days following the accumulation of plaque and is a subclinical lesion which can only be visualized histologically. It is characterized by the increased gingival crevicular fluid, accumulation of Polymorphonuclear Leukocytes (PMNs), and alterations to the connective tissue. Also, alternative pathway of complement activation will lead to augmented production of vasoactive substances coupled with increased vascular permeability and edema. The early lesion occurs within four to seven days following the accumulation of plaque and may exhibit up to $60-70 \%$ of collagen being degraded within the effected zone. The inflammatory infiltrate in the connective tissue is now predominated by lymphocytes, mainly $\mathrm{T}$ cells. Immunological events characterizing the development of gingivitis have been described by Seymour et al. [2]. The T lymphocytes have 2:1 ratio of CD4: CD8 and are activated by expressing high levels of MHC class II antigens. This lesion resembles the features associated with a delayed type hypersensitivity reaction.

The established lesion occurs one to three weeks following plaque accumulation and is characterized by a lymphocytic infiltrate predominated by B cells. Increased permeability of the pocket epithelium permits the inflow of microbial products resulting in high levels of inflammatory mediators such as IL-1, Tumor Necrosis Factor- $\alpha$ (TNF- $\alpha$ ), and prostaglandin $\mathrm{E}_{2}\left(\mathrm{PGE}_{2}\right)$. This lesion may remain stable, or in susceptible in patients due to genetic or environmental factors, and could progress to the advanced lesion. The advanced lesion is characterized by an abundance of plasma cells. In addition, fibroblasts, epithelial cells and macrophages are activated in this environment and will produce Matrix Metalloproteinases (MMP) that degrade the extracellular matrix resulting in the fragmentation of collagen molecules. Clinically, this lesion will result in the loss of connective tissue and alveolar bone and will be diagnosed as periodontitis.

\section{Th1 and Th2 cells}

During the maturation of host response, if the initial lesion is not included by the innate response and the influx of PMNs, then the transition to a $\mathrm{T}$ helper cell predominated lesion will signal the activation of the adaptive arm of the immune 
response. The classic study by Mosmann et al. defined CD4+ helper $\mathrm{T}$ cells based upon their cytokine production as either Th1 or Th2 [3]. According to this paradigm, Th1 cells led to a cellmediated immune response based upon high secretory levels of Interferon- $\gamma($ IFN- $\gamma$ ) and IL-12. In addition, these cytokines influence B cells produce antibody isotypes that enhance antigen uptake and presentation to T cells. Th2 cells promoted a strong humoral response characterized by augmented production of IgE and mast cell activation due to increased production of IL-4, IL5 , and IL-10. Since CD4+ helper T cells have been implicated in playing a critical role in the progression of the periodontal lesion and the transition from gingivitis to periodontitis, several studies have attempted to determine if either Th1 or Th2 played a more critical role in this process $[4,5]$. However, there has been no consensus suggesting that periodontal disease is neither a Th1 nor a Th2 mediated disease. Another perspective suggests that the role of $\mathrm{T}$ lymphocytes in periodontal disease may be one of the immune homeostasis [6].

\section{Th17 and T regulatory cells}

Several additional layers of complexity have been added onto the CD4+ helper $\mathrm{T}$ cell phenotype over the past ten years.The discovery of two additional CD4+ subsets, Th17 and T regulatory cells (Treg), resolved some of the questions which were not adequately explained by the Th1/ Th2 paradigm. Helper $\mathrm{T}$ cell precursors may be driven to differentiate along the Th17 pathway in an environment enriched for Transforming Growth Factor- $\beta$ (TGF- $\beta$ ), IL-1, IL-6, and IL-21. IL-23 has been shown to be critical for Th17 expansion, survival, and pathogenicity [7]. Cytokines produced by Th17 cells, such as IL-21, further promote Th17 development in a manner analogous to IFN- $\gamma$ and IL-4 in the Th1/ Th2 model [8]. An environment deficient in IL-1, IL-6, and IL-21 but with elevated levels of IL-2 would promote differentiation of helper $\mathrm{T}$ cell precursors along the Treg pathway leading to increased production of TGF- $\beta$ and dampening of the inflammatory response. Thus, Th17 and Treg and their respective cytokines are associated with distinct effector functions, inflammation/autoimmunity and immune suppression respectively. In this scenario, the cytokine milieu in which helper $\mathrm{T}$ cells develop plays an important role in selecting the differentiation pathway traversed by the precursor T lymphocyte.

Evidence is accumulating to support the pathogenic role of Th17 cells in inflammatory diseases such as rheumatoid arthritis and periodontitis. An increased level of IL-17 induces the production of inflammatory and osteoclast mediators including TNF- $\alpha$, IL- 1 and IL-6. These cytokines promote the expression of Receptor Activator of Nuclear Factor Kappa-B Ligand (RANKL) on fibroblasts. Activation of RANK on the membrane of the precursor osteoclast following the binding to RANKL is critical for osteoclast formation and bone resorption. An animal study supports the role of IL-17 and Th17 in gingival inflammation and subsequent destruction of alveolar bone in periodontitis [9]. There is also evidence of the presence of IL-17 and Th17 cells in human periodontal lesions which may be associated with

\section{increasing disease severity [10].}

$\mathrm{T}$ lymphocytes are the critical players in the progression of periodontal disease. Disease susceptibility may be regulated by the factors which modulates the individual host response such as genetics and the local environment within the oral cavity. Host response modifiers which attenuate local inflammation in the periodontium, promotes resolution which may be effective as adjunctive therapeutics in the treatment of periodontal disease. A number of human studies have shown that vitamin D and/or calcium intake resulted in diminished alveolar bone loss, reduced gingival inflammation, and less loss of periodontal attachment $[11,12]$. Interestingly, an in vitro study has also reported that 1 , 25- $[\mathrm{OH}]_{2} \mathrm{D}_{3}$ can inhibit monocyte expression of inflammatory mediators including IL-1, IL-6, IL-8, IL-12 and TNF- $\alpha$ [13]. Many of these cytokines are required for establishing an environment that may lead to the development and expansion of Th17. Indeed, it has been postulated that vitamin D may help to "shape" the adaptive immune response by selectively stimulating specific T-helper cell subsets while inhibiting the Th17 pathway [14]. Furthermore, Th17 cells have been shown to be sensitive to 1,25- $[\mathrm{OH}]_{2} \mathrm{D}_{3}$ - mediated suppression and IL-17 production was ablated via a direct transcriptional mechanism [15]. Thus, vitamin $\mathrm{D}$ intake and the serum levels of $1,25-[\mathrm{OH}]_{2} \mathrm{D}_{3}$ may play an important role in determining host susceptibility by selectively promoting a specific T-helper cell subset and their specific cytokines.

\section{Polymicrobial Genetic Dysbiosis: Evolution of a New Paradigm}

Our understanding of the etiology and pathogenesis of periodontal disease, one of the most common chronic inflammatory human diseases, has changed dramatically over the past century. Many theories have been developed to explain periodontal disease pathogenesis, including the 'Traditional and Updated Non-Specific Plaque Hypothesis (T- and U-NSPH)', the 'Specific Plaque Hypothesis (SPH)', the 'Ecological Plaque Hypothesis (EPH)', the role of "red complex" periodontopathogens and the 'Keystone Pathogen Hypothesis (KPH)' [16]. These hypotheses suggest that destructive periodontal disease occurs when: (i) the overall bacterial challenge (from bacteria of similar 'T-NSPH' or variable virulence 'U-NSPH') surpasses the ability of the host to neutralize them, [ii] ecological stresses create a microbial pathogenic shift imbalance, (iii) the ratio of one or more of the 'red complex' bacteria (Porphyromonas gingivalis, Treponema denticola, Tannerella forsythia) increases, or (iv) different low-abundance keystone pathogens manipulate the commensal microbiota to cause disease [16], but fail to integrate the microbial variability with the role of host-specific genetic factors in the impaired immune response.

Recent advances in molecular biology and the 'omics' technologies suggest a more new complex model of periodontal disease pathogenesis, where disease results from Polymicrobial Synergy and Dysbiosis in a susceptible host (PSD model, also associated with inflammatory bowel disease, colon cancer etc) $[17,18]$. According to the PSD model, the host-microbe 
homeostasis (state of equilibrium/ health between the host and the commensal microorganisms) transitions to destructive periodontal disease when the microbiota becomes dysbiotic in a susceptible host $[17,18]$. In this model, no single or few specific pathogens, but a broadly-based dysbiotic, synergistic, microbiota forms periodontal dysbiotic microbial communities, where microbes play specific roles in periodontal disease pathogenesis as keystone pathogens, pathobionts and accessory pathogens [17]. Keystone pathogens can subvert the immune response, orchestrate the shift of the commensal microbiota into dysbiotic, and trigger periodontal destruction. A keystone pathogen example is the low-abundance $P$. gingivalis, which impairs host defense and promotes the growth of the entire microbial community through multiple mechanisms (i.e, interference between complement and Toll-like receptors crosstalk) [19]. Interestingly, $P$. gingivalis is also incriminated as a trigger in the breakdown of immune tolerance by citrullination of host proteins with its Peptidylarginine Deiminase (PAD) enzyme in rheumatoid arthritis susceptible individuals carrying the $H L A$ DRB1 epitope allele [20]. P. gingivalis disrupts the host immune response, inhibits the production of multiple chemokines including the Th1-cell-biasing chemokines IP-10 (CXCL10), Mig (CXCL9) and ITAC (CXCL11) creating a local 'chemokine paralysis' [19-21], and consequently suppresses Th1-dependent cell-mediated immune response. In addition, $P$. gingivalis-induced dysregulation of the host cytokine production includes favoring a pattern with IL-6, IL-23 and especially IL-1 $\beta$ that promotes CD4+ Th17 polarization and Th17- mediated inflammation [22]. The impaired host defense allows pathobionts (i.e. Filifactor alocis) a previously unrecognized pathogen [23] to trigger destructive inflammation and bone loss, involving both innate and adaptive immune elements [17]. Accessory pathogens, commensal in health, enhance the virulence of other organisms, as Streptococcus gordonii which facilitates the colonization and increases the virulence of $P$. gingivalis, resulting in more bone loss compared with either microorganism alone [18].

Dysbiotic microbial gene expression and host genetic susceptibility are both crucial in the periodontal disease pathogenesis paradigm. Firstly, polymicrobial synergy involves interbacterial growth facilitation and complex interbacterial signaling, where variable dysbiotic microorganism communities contribute as a group the genes necessary for periodontal disease progression. More specifically, transcriptomics analyses of the human oral microbiome during health and periodontal disease reveal upregulation of mostly consistent gene combinations expressed in destructive periodontal disease, sometimes from organisms not considered major periodontal pathogens [24]. Secondly, destructive periodontal disease initiation requires a susceptible host with multiple potential disease-modifying genes that may influence bacterial selection by attachment and growth, and alter the immune host response [25]. The evolution of the periodontal disease pathogenesis paradigm to a Polymicrobial Genetic Dysbiosis model includes the polymicrobial synergy in a specific environment, the microbial dysbiotic gene expression and the host genetic susceptibility to encompass the complexity of the periodontal diseases.

\section{Conclusion}

Precursor helper T cells may differentiate along a number of different pathways depending upon environmental factors and genetics, reflecting plasticity embedded within this scenario. In a periodontium bathed in cytokines including IL-1, IL-6, IL-21, IL23 , and TGF- $\beta$, in a vitamin D deficient host, or in the presence of a biofilm with elevated levels of the periodontal keystone pathogen P. gingivalis, the Th17 pathway will predominate. Therapeutics which target attenuation of IL-17 production in gingival tissue may prove efficacious as adjunctive aids in the treatment of periodontal disease and in the prevention of disease recurrence.

\section{References}

1. Page RC, Schroeder HE. Pathogenesis of inflammatory periodontal disease. A summary of current work. Lab Invest. 1976;34(3):235-249.

2. Seymour GJ, Gemmell E, Walsh LJ, Powell RN. Immunohistological analysis of experimental gingivitis in humans. Clin Exp Immunol. 1988;71(1):132-137.

3. Mosmann TR, Cherwinski H, Bond MW, Giedlin MA, Coffman RL. Two types of murine helper T cell clone. I. Definition according to profiles of lymphokine activities and secreted proteins. J Immunol. 1986;136(7):2348-2357.

4. Gemmell E, Seymour GJ. Immunoregulatory control of Th1/Th2 cytokine profile in periodontal disease. Periodontal 2000. 2004;35:2141.

5. Taubman MA, Valverde $\mathrm{P}$, Han $\mathrm{X}$, Kawai T. Immune response: the key to bone resorption in periodontal disease. J Periodontol. 2005;76(11):2033S-2044S.

6. Gemmell E, Marshall RI, Seymour GJ. Cytokines and prostaglandins in immune homeostasis and tissue destruction in periodontal disease. Periodontal 2000. 1997;14:112-143.

7. Stockinger B, Veldohen M. Differentiation and function of Th17 T cells. Curr Opin Immunol. 2007;19(3):281-286.

8. Korn T, Bettelli E, Gao W, Awasthi A, Jäger A, Strom TB, et al. IL-21 initiates an alternative pathway to induce proinflammatory $\mathrm{T}(\mathrm{H}) 17$ cells. Nature. 2007;448(7152):484-487.

9. Eskan MA, Jotwani R, Abe T, Chmelar J, Lim JH, Liang S, et al. The leukocyte integrin antagonist Del-1 inhibits IL-17-mediated inflammatory bone loss. Nature Immunology. 2012;13(5):465-473. doi: $10.1038 /$ ni. 2260

10. Okui T, Aoki Y, Ito H, Honda T, Yamazaki K. The presence of IL17+/FOXP3+ double-positive cells in periodontitis. J Dent Res. 2012;91(6):5714-579. doi: 10.1177/0022034512446341.

11. Dietrich T, Joshipura KJ, Dawson-Hughes B, Bischoff-Ferrari HA. Association between serum concentrations of 25-hydroxyvitamin D3 and periodontal disease in the US population. Am J Clin Nutr. 2004;80(1):108-113.

12. Hildebolt CF. Effect of vitamin D and calcium on periodontitis. J Periodontol. 2005;76(9):1576-1587.

13. Giulietti A, van Etten E, Overbergh L, Stoffels K, Bouillon R, Mathieu C. Monocytes from type II diabetic patients have a pro-inflammatory profile. 1,25-dihydroxyvitamin D3 works as an anti-inflammatory. Diabetes Res Clin Prac. 2007;77(1):47-57. 
14. Stein SH, Livada R, Tipton DA. Re-evaluating the role of vitamin D in the periodontium. J Periodont Res. 2014; 49(5):545-553. doi: 10.1111/jre.12149.

15. Joshi S, Pantalena LC, Liu XK, Gaffen SL, Liu H, Rohowsky-Kochan C, et al. 1,25-dihydroxyvitamin D3 ameliorates Th17 autoimmunity via transcriptional modulation of IL-17A. Mol Cell Biol. 2011;31(17):36533669. doi: 10.1128/MCB.05020-11.

16. Rosier BT, De Jager M, Zaura E, Krom BP. Historical and contemporary hypotheses on the development of oral diseases: are we there yet? Front Cell Infect Microbiol. 2014;4:92. doi: 10.3389/fcimb.2014.00092.

17. Hajishengallis G. Immunomicrobial pathogenesis of periodontitis: keystones, pathobionts, and host response. Trends Immunol. 2014;35(1):3-11. doi: 10.1016/j.it.2013.09.001.

18. Lamont RJ, Hajishengallis G. Polymicrobial synergy and dysbiosis in inflammatory disease. Trends Mol Med. 2015;21(3):172-83. doi: 10.1016/j.molmed.2014.

19. Hajishengallis G, Lamont RJ. Breaking bad: manipulation of the host response by Porphyromonas gingivalis. Eur J Immunol. 2014; 44(20):328-338. doi: 10.1002/eji.201344202.

20. Hajishengallis G. Periodontitis: from microbial immune subversion to systemic inflammation. Nat Rev Immunol. 2015;15(1):30-44. doi: $10.1038 / \mathrm{nri} 3785$

21. Darveau RP, Belton CM, Reife RA, Lamont RJ. Local chemokine paralysis, a novel pathogenic mechanism for Porphyromonas gingivalis. Infect Immun. 1998; 66(4):1660-1665.

22. Moutsopoulos NM, Kling HM, Angelov N, Jin W, Palmer RJ, Nares S, et al. Porphyromonas gingivalis promotes Th17 inducing pathways in chronic periodontitis. J Autoimmun. 2012;39:294-303. doi: 10.1016/j. jaut.2012.03.003.

23. Griffen AL, Beall CJ, Campbell JH, Firestone ND, Kumar PS, Yang ZK, et al. Distinct and complex bacterial profiles in human periodontitis and health revealed by 16 S pyrosequencing. ISME J. 2012;6:1176-85. doi: 10.1038/ismej.2011.191.

24. Duran-Pinedo AE, Chen T, Teles R, Starr JR, Wang X, Krishnan K, et al. Community-wide transcriptome of the oral microbiome in subjects with and without periodontitis. ISME J. 2014;8(8):1659-1672. doi: 10.1038/ismej.2014.23.

25. Nibali L, Henderson B, Sadiq ST, Donos N. Genetic dysbiosis: the role of microbial insults in chronic inflammatory diseases. J Oral Microbiol. 2014;6:22962. doi: 10.3402/jom.v6.22962. 